University of South Carolina

Scholar Commons

3-2009

\title{
Electronic Media Exposure and Its Association With Activity- Related Outcomes in Female Adolescents: Cross-Sectional and Longitudinal Analyses
}

\author{
Felipe Lobelo \\ Marsha Dowda \\ University of South Carolina - Columbia, mdowda@mailbox.sc.edu \\ Karin A. Pfeiffer \\ Russell R. Pate \\ University of South Carolina - Columbia, rpate@mailbox.sc.edu
}

Follow this and additional works at: https://scholarcommons.sc.edu/

sph_physical_activity_public_health_facpub

Part of the Public Health Commons

Publication Info

Published in Journal of Physical Activity and Health, Volume 6, Issue 2, 2009, pages 137-143.

Lobelo, F., Dowda, M., Pfeiffer, K. A., Pate, R. R. (2009). Electronic media exposure and its association with activity-related outcomes in female adolescents: Cross-sectional and longitudinal analyses. Journal of Physical Activity and Health, 6(2), 137-143.

(c) Journal of Physical Activity and Health, 2009, Human Kinetics

This Article is brought to you by the Physical Activity and Public Health at Scholar Commons. It has been accepted for inclusion in Faculty Publications by an authorized administrator of Scholar Commons. For more information, please contact digres@mailbox.sc.edu. 


\title{
Electronic Media Exposure and Its Association With Activity-Related Outcomes in Female Adolescents: Cross-Sectional and Longitudinal Analyses
}

\author{
Felipe Lobelo, Marsha Dowda, Karin A. Pfeiffer, and Russell R. Pate
}

\begin{abstract}
Background: Few investigations have assessed in adolescent girls the cross-sectional and longitudinal associations between elevated exposure to electronic media (EM) and activityrelated outcomes such as compliance with physical activity (PA) standards or cardiorespiratory fitness (CRF). Methods: Four-hundred thirty-seven white and African American girls were assessed at the 8th, 9th, and 12th grades. PA and EM (TV/video watching, electronic games, Internet use) were self-reported, and CRF was estimated using a cycle-ergometer test. Hi EM exposure was defined as $\geq$ four 30-minute blocks/d. Results: 8th-, 9th-, and 12th-grade girls in the Hi EM group showed lower compliance with PA standards and had lower CRF than the Low EM group $(P \leq .03)$. Girls reporting Hi EM exposure at 8th and 9th grades had lower vigorous PA and CRF levels at 12th grade than girls reporting less EM exposure $(P \leq .03)$. Conclusion: Girls reporting exposure to EM for 2 or more hours per day are more likely to exhibit and maintain low PA and CRF levels throughout adolescence. These results enhance the scientific basis for current public health recommendations to limit adolescent girls' daily exposure to television, electronic games, and Internet use to a combined maximum of 2 hours.
\end{abstract}

Keywords: television watching, physical fitness, physical activity, prospective study, adolescent girls

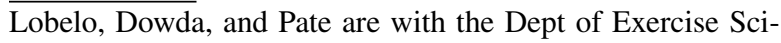
ence, Arnold School of Public Health, University of South Carolina, Columbia, SC 29208. Pfeiffer is with the Dept of Kinesiology, Michigan State University, East Lansing, MI 48824.
Reduced physical activity (PA) and increased sedentary time have been associated with health risks in pediatric ${ }^{1,2}$ and adult populations, ${ }^{3,4}$ and both are thought to independently contribute to increased adiposity ${ }^{5,6}$ and metabolic risk in children and adolescents. ${ }^{7}$

Researchers have used television (TV) watching and other exposure to electronic media (EM) as a surrogate for time spent in sedentary activity. Elevated exposure to EM has been associated with decreased participation in PA, obesity, low cardiorespiratory fitness (CRF), and other negative health outcomes. ${ }^{1,6-8}$ The specific mechanisms underlying these relationships have not been established, and although researchers have proposed that EM displaces physically active pursuits, studies addressing this hypothesis have presented conflicting results. ${ }^{9-11}$ These inconsistencies might reflect the inherent difficulty in measuring PA and use of EM. ${ }^{12}$

The factors that influence PA and sedentary behaviors in adolescents differ among gender and race groups. ${ }^{13,14}$ Although some research indicates that adolescent boys might participate in recommended levels of moderate-to-vigorous PA even after devoting significant amounts of time to sedentary activities, the association between PA and sedentary activity in adolescent girls is not clear. ${ }^{15}$ In addition, most investigations exploring the health consequences of elevated exposure to EM have looked only at TV watching, have focused on weight status/body composition outcomes, and have relied on single cross-sectional measurements. ${ }^{12}$ Few investigations have used prospective designs to examine the effects of comprehensive EM indices on adiposity outcomes in ethnically diverse samples of adolescent girls. ${ }^{16-18}$ In addition, the existing prospective studies have not assessed the effects of elevated EM exposure on other important health outcomes, such as future compliance with PA recommendations and CRF.

The information derived from a prospective study addressing the effects of elevated EM exposure on activity-related outcomes could inform the design of interventions tailored to decrease specific levels of EM 
exposure, if they are found to be detrimental to such outcomes in adolescent girls. Such information would be of considerable public health relevance because PA and CRF levels decline sharply during adolescence, ${ }^{5}$ especially among girls, track from childhood into adulthood, ${ }^{8}$ and constitute independent predictors of cardio-metabolic disease risk. $^{7}$ In addition, the ubiquitous access and exposure that adolescents have to a variety of forms of EM entertainment, not just television, makes it important to study the effects of EM using comprehensive EM indexes. Accordingly, the purpose of this study was to examine, using both crosssectional and longitudinal analyses, the relationship between elevated exposure to EM (including TV/video watching, electronic games, and Internet use) and compliance with PA standards and CRF levels in African American and white adolescent girls followed over a 4year period.

\section{Methods}

\section{Participants and Data Collection}

This investigation followed a cohort of girls prospectively from 8th through 12th grade, as part of a schoolbased PA intervention study known as LEAP (Lifestyle Education for Activity Program). ${ }^{19}$ Two successive grade cohorts of girls were measured. Baseline data were collected when the girls were in 8th grade, and follow-up data were collected when they were in 9th and 12th grades. Trained data collectors used standardized protocols and scripts when administering the measures to participants in groups of 20 to 30 girls. The procedures were approved by the University of South Carolina Institutional Review Board. Each participant and her parent or guardian provided written informed consent.

Overall, 2744 girls (average age of 13.6 [0.6] years; 48.7\% African American) completed the measurement protocol in 8th grade and were invited to complete the protocol again in 9th and 12th grades. For the purposes of the current investigation, complete data were available for 2101 girls in 8th grade, 1685 girls in 9th grade, and 1424 girls in 12th grade for the cross-sectional analyses. Longitudinal cohort analyses were based on the 437 girls (15.9\% of the initial sample) who had complete data on EM exposure, PA, and CRF at the 8th-, 9th-, and 12th-grade time points. This sample had the same age at baseline (average age 13.6 [0.6] years) as the larger study sample. In addition, CRF, vigorous PA, and moderate-to-vigorous PA did not differ between the 2 samples at baseline. A higher percentage of girls included in the longitudinal cohort analysis were African American (58\%).

\section{Measurement of Physical Activity}

PA was assessed using the 3-Day Physical Activity Recall (3DPAR). A complete description of the 3DPAR has been published elsewhere. ${ }^{20}$ Briefly, the instrument was completed on a Wednesday, and participants recalled their PA behavior on the previous 3 days of the week ( 2 weekdays and 1 weekend day), beginning with the most recent day. The instrument includes a list of common activities grouped into broad categories to improve activity recall. For every 30-minute time increment (block), students reported the main activity performed and then rated its relative intensity. Each activity was assigned a metabolic equivalent (MET) using the Compendium of Physical Activities. ${ }^{21}$ Data from each day were reduced to the number of blocks of moderateto-vigorous PA ( $\geq 3.0$ METs; MVPA) and vigorous physical activity ( $\geq 6$ METs; VPA), and an average per day was calculated for each of these variables over the 3 days. The 3DPAR has been shown to be a valid and reliable tool for assessing moderate, vigorous, and total PA in African American and white adolescent girls based on correlations with an objective measure of PA derived from accelerometry $(r=.42)^{20}$ and a self-report measure of sport involvement. ${ }^{22}$ Girls who reported participating in an average of 1 or more blocks of VPA per day or 2 or more blocks of MVPA per day, over the 3-day reporting period, were considered to meet PA standards for adolescents. ${ }^{23,24}$

\section{Measurement and Classification of Electronic Media Exposure}

The design and psychometric characteristics of the 3DPAR allow researchers to collect information about activities at the lower end of the intensity continuum (such as EM), as well as activities of moderate or vigorous intensity. ${ }^{20,25}$ The 3DPAR asks about use of EM in a manner similar to other instruments designed to measure sedentary activities. ${ }^{26,27}$ The total number of 30minute time blocks for which girls reported TV/video watching, electronic games, and Internet use on the 3DPAR was used as an index of EM exposure. Data were reduced to the number of blocks per day in which the subjects reported EM as their main activity. The recommendations of the Committee on Public Education of the American Academy of Pediatrics ${ }^{28}$ and Objective 22-11 of Healthy People $2010^{29}$ regarding the use of EM informed our selection of a threshold to define high exposure to EM. For the cross-sectional analyses, girls in each grade were classified in 1 of 2 exposure groups. Girls in the low electronic media (Low EM) exposure group were those who reported an average of fewer than 4 blocks of EM per day. Girls in the high electronic media (Hi EM) exposure group reported an average of 4 or more blocks of EM per day, at each of the 3 grade levels. In addition, we evaluated multiple categories $(\leq 2,2$ to $3, \geq 4$ blocks per day) of exposure to EM to try to identify dose-response associations that could have public health implications in terms of EM exposure recommendations.

For the longitudinal analyses, 12th-grade outcomes (PA, CRF) were predicted based on the EM group clas- 
sification at the 8th- or 9th-grade assessment, 8th-grade data were used for the 8th- to 12th-grade analysis, and 9th-grade data for the 9th- to 12th-grade analysis. We also defined the EM groups based on the consistency of exposure over 2 years (combined 8th and 9th grade). Accordingly, the Combined Low EM exposure group included girls who reported fewer than 4 blocks of EM per day at either 8th or 9th grade or both. The Combined Hi EM exposure group included girls who reported an average of 4 or more blocks of EM per day at both the 8th- and 9th-grade assessments.

\section{Cardiorespiratory Fitness Measure}

A modified version of the Physical Work Capacity 170 test was used to obtain an estimate of CRF. ${ }^{30}$ The test involves performing graded, submaximal exercise on a cycle ergometer for three, 2-minute stages. Heart rate (HR) was recorded as the subject performed 3 submaximal power outputs that approximated 120,150 , and 180 beats/min. The goal of the protocol was to elicit a HR of at least 160 beats/min by the end of the third stage; otherwise, subjects completed a fourth stage. CRF was estimated as the power output corresponding to a heart rate of 170 beats/min using a linear regression of HR versus power output computed for each participant and expressed as $\mathrm{kgm} \cdot \mathrm{min}^{-1} \cdot \mathrm{kg}^{-1}$ body weight.

\section{Statistical Analysis}

Descriptive statistics were calculated for selected variables by EM group classification. Analysis of the crosssectional association between EM exposure and percent meeting PA standards (logistic regression) and CRF (2way ANOVAs) was performed using mixed models. Each model included race, EM group, and the interaction between race and EM group and controlled for the effects of the LEAP intervention (control/intervention status), age, and body mass index (BMI). School was treated as a random variable. For the longitudinal analyses, mixed models were used to predict 12th-grade outcomes based on either 8th, 9th, or combined 8th and 9th EM exposure data while adjusting for socioeconomic status (only available at 12th grade), age, and BMI. The impact of EM exposure and race on PA and CRF was assessed using a significance level $(P$ value $) \leq .05$ for main effects and $\leq .10$ for interactions. Data analysis was conducted using SAS version 8.2. ${ }^{31}$

\section{Results}

Few girls $(\mathrm{n}=20)$ from race/ethnic groups other than African American or white participated in the study; therefore, we report only the results for these 2 race groups. The effect of the LEAP intervention (control/ intervention status) was tested in all analyses and was never significant $(P>.05)$. Table 1 shows the demographic and anthropometric characteristics and the EM and PA levels of the study sample by assessment year.
Table 1 Demographic Characteristics, BMI, EM and PA Levels of Adolescent Girls at 3 Assessment Points

\begin{tabular}{lc}
\hline Characteristics & Mean (SD) or \% \\
\hline 8th grade $(\mathrm{n}=2101)$ & $13.6(0.6)$ \\
Age $(\mathrm{y})$ & $23(5.4)$ \\
BMI $\left(\mathrm{kg} / \mathrm{m}^{2}\right)$ & 51.9 \\
$\%$ African American & 41.9 \\
$\%$ Hi EM exposure group & 42.1 \\
$\%$ Meeting VPA standard & 70.7 \\
$\%$ Meeting MVPA standard & \\
9th grade $(\mathrm{n}=1685)$ & $23.6(5.6)$ \\
Age (y) & 49.3 \\
BMI $\left(\mathrm{kg} / \mathrm{m}^{2}\right)$ & 36.0 \\
$\%$ African American & 41.2 \\
$\%$ Hi EM exposure group & 71.6 \\
$\%$ Meeting VPA standard & \\
$\%$ Meeting MVPA standard & $17.7(0.6)$ \\
12th grade (n = 1424) & $25(6.2)$ \\
Age (y) & 55.8 \\
BMI (kg/m $\left.{ }^{2}\right)$ & 30.1 \\
$\%$ African American & 35.6 \\
$\%$ Hi EM exposure group & 74.9 \\
$\%$ Meeting VPA standard &
\end{tabular}

Abbreviations: BMI, body mass index; EM, electronic media; PA, physical activity; VPA, vigorous physical activity; MVPA, moderateto-vigorous physical activity; Hi EM, reported $\geq$ four 30-min blocks of EM per day; VPA standard, reported $\geq 0$ ene 30-min block of VPA per day; MVPA standard, reported $\geq$ two 30-min blocks of MVPA per day.

\section{Cross-Sectional Associations Between Electronic Media Exposure and Activity- Related Outcomes}

At the 8th-, 9th-, and 12th-grade assessments, white and African American girls in the Hi EM exposure group had lower compliance with the VPA and MVPA standards compared with girls in the Low EM exposure group $(P \leq .016)$. Regardless of EM group classification, the proportion of girls in the 8th, 9th, and 12th grade meeting the VPA and MVPA standards was lower among African American than white girls $(P \leq .004$; Table 2).

At 8th grade, white girls in the Hi EM exposure group had lower CRF $\left(\mathrm{kgm} \cdot \mathrm{min}^{-1} \cdot \mathrm{kg}^{-1}\right)$ than white girls in the Low EM exposure group $(\mathrm{EM} \times$ Race interaction $P=.07)$. At 9 th and 12 th grades, white and African American girls in the Hi EM exposure group had lower CRF than girls in the Low EM exposure group ( $P$ $\leq .023$ ). Regardless of EM group classification, African American girls had lower CRF than white girls in the 9 th and 12 th grades $(P<.001$; Table 2$)$. 
Table 2 Cross-Sectional Associations Between Exposure to Electronic Media and ActivityRelated Outcomes Among Adolescent Girls at 3 Assessment Points

\begin{tabular}{|c|c|c|c|c|c|c|c|}
\hline \multirow[b]{2}{*}{ Variable } & \multicolumn{2}{|c|}{ Low EM ${ }^{a}$} & \multicolumn{2}{|c|}{$\mathrm{Hi} \mathrm{EM}^{\mathrm{b}}$} & \multicolumn{3}{|c|}{ Effects $^{c}$} \\
\hline & $\begin{array}{c}\text { White } \\
\text { mean (SE) } \\
\text { or } \%\end{array}$ & $\begin{array}{c}\text { AA } \\
\text { mean (SE) } \\
\text { or } \%\end{array}$ & $\begin{array}{c}\text { White } \\
\text { mean (SE) } \\
\text { or } \%\end{array}$ & $\begin{array}{c}\text { AA } \\
\text { mean (SE) } \\
\text { or } \%\end{array}$ & $\begin{array}{c}\text { EM } \\
P\end{array}$ & $\begin{array}{c}\text { Race } \\
P\end{array}$ & $\begin{array}{c}\mathrm{EM} \times \text { Race } \\
\boldsymbol{P}\end{array}$ \\
\hline \multicolumn{8}{|l|}{$\geq 1$ block/d VPA } \\
\hline 8 th grade $(n=2101)$ & $54.7(2.4)$ & $40.4(2.4)$ & $42.1(2.6)$ & $28.4(2.3)$ & $<.001$ & $<.001$ & .98 \\
\hline 9 th grade $(\mathrm{n}=1685)$ & $48.8(2.6)$ & $37.2(2.7)$ & $41.3(3.4)$ & $34.4(3.1)$ & .016 & $<.001$ & .34 \\
\hline 12 th grade $(n=1388)$ & $44.9(2.4)$ & $35.5(2.3)$ & $33(3.9)$ & $21.4(3.1)$ & $<.001$ & $<.001$ & .69 \\
\hline \multicolumn{8}{|l|}{$\geq 2$ blocks/d MVPA } \\
\hline 8 th grade $(n=2101)$ & $84.9(2.1)$ & $69.1(2.3)$ & $69.2(2.8)$ & $56.5(2.2)$ & $<.001$ & $<.001$ & .45 \\
\hline 9 th grade $(n=1685)$ & $81.4(1.9)$ & $68.8(2.1)$ & $70.8(2.8)$ & $59.3(2.4)$ & $<.001$ & $<.001$ & .81 \\
\hline 12 th grade $(n=1388)$ & $84.7(2.2)$ & $77.1(2.1)$ & $67.1(3.5)$ & $59.3(2.7)$ & $<.001$ & .004 & .97 \\
\hline \multicolumn{8}{|l|}{$\mathrm{CRF}\left(\mathrm{kgm} \cdot \mathrm{min}^{-1} \cdot \mathrm{kg}^{-1}\right)$} \\
\hline 8 th grade $(n=1954)$ & $11.9(0.2)$ & $11.5(0.2)$ & $11.3(0.2)$ & $11.4(0.2)$ & .032 & .41 & .07 \\
\hline 9 th grade $(n=1601)$ & $12.7(0.2)$ & $11.9(0.2)$ & $11.6(0.3)$ & $11.2(0.2)$ & $<.001$ & $<.001$ & .21 \\
\hline 12 th grade $(n=908)$ & $12.4(0.3)$ & $11(0.3)$ & $11.6(3.9)$ & $10.6(0.3)$ & .023 & $<.001$ & .39 \\
\hline
\end{tabular}

Abbreviations: EM, electronic media; PA, physical activity; AA, African American; VPA, vigorous physical activity; MVPA, moderate-to-vigorous physical activity; CRF, cardiorespiratory fitness. Estimates adjusted by age and body mass index.

${ }^{a}$ Reported $<$ four 30-min blocks of EM per day.

${ }^{\mathrm{b}}$ Reported $\geq$ four 30-min blocks of EM per day.

c Bold values indicate statistically significant main effect at $P<.05$ and significant interaction effects at $P<.10$.

Furthermore, the evaluation of multiple categories of exposure to EM showed an inverse association between EM (categorized into 3 exposure groups) and all the activity-related outcomes (MVPA, VPA, fitness). However, when comparing estimates for the $3 \mathrm{EM}$ groups, the first 2 ( $\leq 2$ and 2 to 3 blocks per day) did not differ but the last group ( $\geq 4$ blocks per day) was consistently different from the first 2 EM groups, indicating a possible threshold effect at that level of EM exposure. For example, the proportions of 8th graders meeting the MVPA standard by the 3 EM exposure groups were 69\%, 69\%, and 57\% for African American girls and $87 \%, 84 \%$, and $68 \%$ for white girls. Furthermore, BMI $(P=.80)$, age $(P=.62)$, and the interaction between race and $\operatorname{EM}(P=.36)$ were not significant.

\section{Longitudinal Associations Between Electronic Media Exposure and Activity- Related Outcomes}

Girls classified in the Hi EM exposure group at both 8th and 9 th grades combined had lower compliance with the VPA standard at 12th grade than girls who reported less exposure to $\mathrm{EM}$ in those grades $(P=.027$; Table 3$)$. Among white girls, a report of Hi EM at either 8th or 9th grade or at both 8th and 9th grades combined predicted lower compliance with the MVPA standard at 12th grade (all EM $\times$ Race interaction $P \leq .07$; Table $3)$.

$\mathrm{CRF}$ at 12th grade was lower for girls in the Hi EM versus Low EM groups at 8th grade $(P=.045)$, and this difference was greater for white than for African Ameri- can girls (race effect $P=.023$ ). White girls in the Hi EM group at 9th grade had lower CRF at 12th grade than girls in the Low EM group at 9th grade $(\mathrm{EM} \times$ Race interaction $P=.015$ ). In addition, girls reporting Hi EM at both 8th and 9th grades combined showed lower CRF at 12 th grade than girls who reported less exposure to EM in those grades $(P=.023)$, and this difference was greater for white than for African American girls (race effect $P=.04$; Table 3 ).

\section{Discussion}

This investigation assessed the cross-sectional and longitudinal associations between exposure to electronic media and the physical activity and cardiorespiratory fitness levels of a biracial cohort of 13- to 18-year-old girls. The study found an inverse relationship between exposure to EM and compliance with current PA standards, as well as with the CRF levels of African American and white adolescent girls. This relationship was detected in both cross-sectional and longitudinal analyses and suggested a possible threshold effect at an EM exposure level of 4 or more 30-minute blocks per day. These findings contribute to the scientific basis for current public health ${ }^{29}$ and clinical recommendations ${ }^{28}$ to limit adolescents' exposure not only to television but also to videogames and Internet use to a combined maximum of 2 hours per day. The findings of this study suggest that the significance of these public health recommendations goes beyond body composition and extends to important health-related outcomes such as compliance with PA recommendations and CRF. These findings 
Table 3 Predicting Activity-Related Outcomes at 12th Grade Based on Electronic Media Use at 8th or 9th Grade or Combining 8th- and 9th-Grade Datad Among Adolescent Girls

\begin{tabular}{|c|c|c|c|c|c|c|c|}
\hline \multirow[b]{2}{*}{ Variable } & \multicolumn{2}{|c|}{ Low EM ${ }^{a}$} & \multicolumn{2}{|c|}{ Hi EM ${ }^{b}$} & \multicolumn{3}{|c|}{ Effects $^{c}$} \\
\hline & $\begin{array}{c}\text { White } \\
\text { mean (SD) } \\
\text { or } \%\end{array}$ & $\begin{array}{c}\text { AA } \\
\text { mean (SD) } \\
\text { or } \%\end{array}$ & $\begin{array}{c}\text { White } \\
\text { mean (SD) } \\
\text { or } \%\end{array}$ & $\begin{array}{c}\text { AA } \\
\text { mean (SD) } \\
\text { or } \% \\
\end{array}$ & $\begin{array}{c}\text { EM } \\
P\end{array}$ & $\begin{array}{c}\text { Race } \\
P\end{array}$ & $\begin{array}{c}\text { EM } \times \text { Race } \\
P\end{array}$ \\
\hline \multicolumn{8}{|l|}{$\geq 1$ block/day VPA $(n=437)$} \\
\hline 8th grade & $42(4.7)$ & $34.9(4.3)$ & $33(6.9)$ & $29.1(4.9)$ & .15 & .3 & .75 \\
\hline 9th grade & $37.6(4.6)$ & $31.9(4.2)$ & $42.1(6.7)$ & $30.5(4.8)$ & .86 & .07 & .60 \\
\hline $\begin{array}{l}\text { Combining 8th and 9th } \\
\text { grades }^{\mathrm{d}}\end{array}$ & $42(4.4)$ & $35.3(4.1)$ & $26.2(9)$ & $24.4(6.1)$ & .027 & .49 & .68 \\
\hline \multicolumn{8}{|c|}{$\geq 2$ block/day MVPA $(n=437)$} \\
\hline 8th grade & $78.3(4.3)$ & $63(4.2)$ & $68.2(6.5)$ & $71.9(4.4)$ & .90 & .25 & .05 \\
\hline 9th grade & $81.5(4.2)$ & $65.7(3.9)$ & $67.5(6.3)$ & $68.7(4.4)$ & .25 & .13 & .07 \\
\hline $\begin{array}{l}\text { Combining 8th and 9th } \\
\text { grades }^{\mathrm{d}}\end{array}$ & $78.4(4)$ & $65.7(3.6)$ & $59.4(8.6)$ & $71.1(5.7)$ & .24 & .92 & .03 \\
\hline \multicolumn{8}{|c|}{$\begin{array}{l}\mathrm{CRF}\left(\mathrm{kgm} \cdot \mathrm{min}^{-1} \cdot \mathrm{kg}^{-1} ; \mathrm{n}=\right. \\
\text { 320) }\end{array}$} \\
\hline 8th grade & $12.5(0.4)$ & $10.9(0.4)$ & $10.8(0.6)$ & $10.1(0.4)$ & .045 & .023 & .51 \\
\hline 9th grade & $12.5(0.4)$ & $10.7(0.4)$ & $10.7(0.6)$ & $10.3(0.4)$ & .016 & .015 & .08 \\
\hline $\begin{array}{l}\text { Combining 8th and 9th } \\
\text { grades }^{\mathrm{d}}\end{array}$ & $12.3(0.5)$ & $10.9(0.4)$ & $10.2(0.7)$ & $10.3(0.4)$ & .023 & .04 & .45 \\
\hline
\end{tabular}

Abbreviations: EM, electronic media; PA, physical activity; AA, African American; VPA, vigorous physical activity; MVPA, moderate-to-vigorous physical activity; CRF, cardiorespiratory fitness. Estimates adjusted by age, BMI, and socioeconomic status.

${ }^{\text {a }}$ Reported $<$ four 30-min blocks of EM per day.

${ }^{\mathrm{b}}$ Reported $\geq$ four 30-min blocks of EM per day.

${ }^{\mathrm{c}}$ Bold values indicate statistically significant main effect at $P<.05$ and significant interaction effects at $P<.10$.

${ }^{d}$ Low EM: reported <four 30-min blocks of EM per day at either 8th or 9th or at both grades $(n=336)$; Hi EM: reported $\geq$ four 30-min blocks of EM per day at both 8th and 9th grades $(\mathrm{n}=101)$.

also are important given the decline in PA and CRF that girls experience during adolescence, ${ }^{5,8,19}$ placing them at increased risk for the development of early cardiometabolic disease. . $^{23}$

In the current study, we found consistent inverse associations between EM exposure and compliance with VPA and MVPA standards among 8th-, 9th-, and 12th-grade girls. In addition, elevated exposure to EM at both 8th and 9th grades predicted lower compliance with the VPA standard at 12th grade, and in white girls, elevated exposure to EM at either 8th or 9th grade or at both grades combined predicted lower compliance with the MVPA standard at 12th grade. Most previous investigations, including a meta-analysis on the topic, have reported an inverse association between TV exposure and PA outcomes among adolescents. ${ }^{1,2,10,12,32}$ Nevertheless, a study in a cohort of Canadian youth from the early $1980 \mathrm{~s}^{8}$ and one in prepubertal German youth ${ }^{33} \mathrm{did}$ not detect a significant association, probably because their overall TV exposure levels were low. However, current generations of adolescents engage in a high level of sedentary time, if the combined exposure to TV and other common forms of EM such as videogames and computer/Internet use are considered. For example, in a systematic review of 90 studies, girls age 13 to 18 years engaged in, on average, 106 minutes of TV watching, 21 minutes of videogames, and 30 minutes of computer use per day. ${ }^{26}$ If combined, these estimates indicate that the average adolescent girl is engaging in approximately 157 minutes of EM per day, thus exceeding current public health recommendations. These results have substantial implications for public health. First, they confirm that to reduce the risk of low PA, contemporary adolescent girls' daily exposure to EM should be limited to a maximum of 2 hours of combined TV, videogames, and computer use. They also suggest that a persistent high level of exposure to EM during early adolescence constitutes a powerful and independent risk factor for future PA participation among adolescent girls. Therefore, adolescent girls' EM exposure levels should be monitored regularly and adjusted to reduce the risk of low PA during this critical developmental period.

Girls reporting elevated exposure to EM exhibited significantly lower CRF levels in both cross-sectional and longitudinal analyses than did girls reporting less exposure to EM. Previous studies have detected an inverse cross-sectional association between TV watching and CRF in youth., 2,834 Furthermore, results from longitudinal birth cohort analyses showed that exposure to TV watching during childhood and adolescence predicted poor CRF levels in early adulthood. ${ }^{35}$ However, others have reported that leisure-time Internet use was not correlated with CRF among African American 
adolescent girls, ${ }^{36}$ probably because the amount of EM captured by such activity is limited. To our knowledge, our study is the first to investigate the effects of combined exposure to a variety of EM forms and CRF in adolescent girls. The results of the current study are similar to those from previous longitudinal investigations, suggesting an inverse, graded association between EM exposure and future CRF levels. Because a high proportion of girls in the Hi EM group also failed to meet the MVPA standard, the evidence suggests that a possible interaction between this level of EM exposure and insufficient PA promotes a steeper decline in CRF over time. The results of the current investigation extend the validity of current public health recommendations to limit EM exposure to an important health-related outcome, CRF, a powerful and independent correlate of cardiovascular ${ }^{37}$ and metabolic ${ }^{38,39}$ disease risk among adolescents. Low CRF is emerging as an important public health problem among adolescents, ${ }^{40,41}$ and the results of this study indicate that, among girls, the prospective risk of falling into low-fitness categories ${ }^{40}$ increases if EM exposure exceeds recommended levels.

Strengths of this investigation include the prospective study of a diverse sample of adolescent girls and the use of instruments with proven validity and reliability to measure the outcomes of interest. The study used an index of EM exposure that incorporated a variety of sedentary activities, including TV/video watching, videogame playing, and Internet use, which might provide a better representation of the sedentary behaviors of current generations of adolescent girls than TV watching alone. In addition, the approach we used to categorize the EM exposure enabled us to define very distinct groups in terms of EM "dose" for both the crosssectional and longitudinal analyses and allowed us to test the validity of current EM-use recommendations in relation to important health-related outcomes, such as PA and CRF. A limitation of the current study is the possibility of misclassification of PA and EM exposure owing to the self-report nature of the data. In addition, although the sample of girls that completed the measurement protocol at all 3 time points was very comparable to the larger LEAP study sample, we acknowledge that the possibility of selection bias cannot be ruled out completely.

In conclusion, the results of this study indicate that elevated exposure to EM is negatively associated, in a cross-sectional and longitudinal fashion, with compliance with physical activity standards and with the cardiorespiratory fitness levels of adolescent girls. These finding are noteworthy because low PA and CRF levels are strong and independent predictors of cardiometabolic health even during adolescence. . $38,39,41$ Race acted as an effect modifier of some of the relationships we observed between EM exposure and PA and CRF. The deleterious effects of high EM exposure appeared to be more prominent among white girls, but the prevalence of high EM exposure was higher among African American girls, underscoring the importance of consid- ering racial and cultural factors when exploring the effects of and intervening on EM use. These results contribute importantly to the basis for current public health recommendations to limit adolescent girls' daily exposure to not only TV watching but other forms of EM, including electronic games and Internet use, to a combined maximum of 2 hours.

\section{Acknowledgments}

The study was funded by a grant from the National Heart, Lung and Blood Institute (NHLBI) of the National Institutes of Health (NIH; R01 HL057775). The authors thank Stewart Trost, PhD, John Sirard, PhD, LaVerne Shuler, and Janna Borden, MS, for administering the study and Gaye Groover Christmus, MPH, for editing the manuscript. We also thank all of the participants, faculty, and staff of the participating schools, as well as the data-collection staff.

\section{References}

1. Andersen RE, Crespo CJ, Bartlett SJ, Cheskin LJ, Pratt M. Relationship of physical activity and television watching with body weight and level of fatness among children: results from the third National Health and Nutrition Examination Survey. JAMA. 1998;279:938-942.

2. Armstrong CA, Sallis JF, Alcaraz JE, Kolody B, McKenzie TL, Hovell MF. Children's television viewing, body fat, and physical fitness. Am J Health Promot. 1998;12:363-368.

3. Hu FB, Li TY, Colditz GA, Willett WC, Manson JE. Television watching and other sedentary behaviors in relation to risk of obesity and type 2 diabetes mellitus in women. JAMA. 2003;289:1785-1791.

4. Salmon J, Bauman A, Crawford D, Timperio A, Owen $\mathrm{N}$. The association between television viewing and overweight among Australian adults participating in varying levels of leisure-time physical activity. Int J Obes Relat Metab Disord. 2000;24:600-606.

5. Biddle SJ, Gorely T, Marshall SJ, Murdey I, Cameron N. Physical activity and sedentary behaviours in youth: issues and controversies. J R Soc Health. 2004;124:2933.

6. Robinson TN. Television viewing and childhood obesity. Pediatr Clin North Am. 2001;48:1017-1025.

7. Ekelund U, Brage S, Froberg K, et al. TV viewing and physical activity are independently associated with metabolic risk in children: the European Youth Heart Study. PLoS Med. 2006;3:e488.

8. Katzmarzyk PT, Malina RM, Song TMK, Bouchard C. Television viewing, physical activity, and health-related fitness of youth in the Quebec family study. J Adolesc Health. 1998;23:318-325.

9. Eisenmann JC, Bartee RT, Wang MQ. Physical activity, TV viewing, and weight in U.S. youth: 1999 Youth Risk Behavior Survey. Obes Res. 2002;10:379-385.

10. Robinson TN, Hammer LD, Killen JD, et al. Does television viewing increase obesity and reduce physical 
activity? cross-sectional and longitudinal analyses among adolescent girls. Pediatrics. 1993;91:273-280.

11. Kaur H, Choi WS, Mayo MS, Harris KJ. Duration of television watching is associated with increased body mass index. J Pediatr. 2003;143:506-511.

12. Marshall SJ, Biddle SJ, Gorely T, Cameron N, Murdey I. Relationships between media use, body fatness and physical activity in children and youth: a meta-analysis. Int J Obes Relat Metab Disord. 2004;28:1238-1246.

13. Crespo CJ, Smit E, Troiano RP, Bartlett SJ, Macera CA, Andersen RE. Television watching, energy intake, and obesity in US children: results from the Third National Health and Nutrition Examination Survey, 1988-1994. Arch Pediatr Adolesc Med. 2001;155:360-365.

14. Gordon-Larsen P, McMurray RG, Popkin BM. Determinants of adolescent physical activity and inactivity patterns. Pediatrics. 2000;105:E83.

15. Lowry R, Wechsler H, Galuska DA, Fulton JE, Kann L. Television viewing and its associations with overweight, sedentary lifestyle, and insufficient consumption of fruits and vegetables among US high school students: differences by race, ethnicity, and gender. $J$ Sch Health. 2002;72:413-421.

16. Davison KK, Marshall SJ, Birch LL. Cross-sectional and longitudinal associations between TV viewing and girls' body mass index, overweight status, and percentage of body fat. J Pediatr. 2006;149:32-37.

17. Gordon-Larsen P, Adair LS, Popkin BM. Ethnic differences in physical activity and inactivity patterns and overweight status. Obes Res. 2002;10:141-149.

18. Berkey CS, Rockett HR, Gillman MW, Colditz GA. Oneyear changes in activity and in inactivity among 10- to 15 -year-old boys and girls: relationship to change in body mass index. Pediatrics. 2003;111:836-843.

19. Pate RR, Ward DS, Saunders RP, Felton G, Dishman RK, Dowda M. Promotion of physical activity in high school girls: a randomized controlled trial. Am J Public Health. 2005;95:1582-1587.

20. Pate RR, Ross R, Dowda M, Trost SG, Sirard J. Validation of a 3-day physical activity recall instrument in female youth. Pediatr Exerc Sci. 2003;15:257-265.

21. Ainsworth BE, Haskell WL, Whitt MC, et al. Compendium of Physical Activities: an update of activity codes and MET intensities. Med Sci Sports Exerc. 2000;32:S498-S516.

22. Motl RW, Dishman RK, Dowda M, Pate RR. Factorial validity and invariance of a self-report measure of physical activity among adolescent girls. Res $Q$ Exerc Sport. 2004;75:259-271.

23. Strong WB, Malina RM, Blimkie CJ, et al. Evidence based physical activity for school-age youth. J Pediatr. 2005;146:732-737.

24. Biddle S, Sallis JF, Cavill NA. Young and Active? Young People and Health Enhancing Physical Activity. Evidence and Implication. London, UK: Health Education Authority; 1998.

25. Dowda M, Pate RR, Felton GM, et al. Physical activities and sedentary pursuits in African American and Caucasian girls. Res Q Exerc Sport. 2004;75:352-360.
26. Marshall SJ, Gorely T, Biddle SJ. A descriptive epidemiology of screen-based media use in youth: a review and critique. J Adolesc. 2006;29:333-349.

27. Schmitz KH, Harnack L, Fulton JE, et al. Reliability and validity of a brief questionnaire to assess television viewing and computer use by middle school children. $J$ Sch Health. 2004;74:370-377.

28. American Academy of Pediatrics, Committee on Public Education. Children, adolescents and television. Pediatrics. 2001;107:423-426.

29. US Department of Health and Human Services. Healthy People 2010. 2nd ed. Washington, DC: US Government Printing Office; 2000.

30. Bengtsson $\mathrm{C}$. The working capacity in normal children, evaluated by submaximal exercise on the bicycle ergometer and compared with adults. Acta Med Scand. 1956;154:91-109.

31. SAS Institute. SAS/STAT User's Guide, Version 8. Cary, NC: SAS Institute, Inc.; 1999.

32. Pate RR, Heath GW, Dowda M, Trost SG. Associations between physical activity and other health behaviors in a representative sample of US adolescents. Am J Public Health. 1996;86:1577-1581.

33. Grund A, Krause H, Siewers M, Rieckert H, Muller MJ. Is TV viewing an index of physical activity and fitness in overweight and normal weight children? Public Health Nutr. 2001;4:1245-1251.

34. Tucker LA. The relationship of television viewing to physical fitness and obesity. Adolescence. 1986;21:797806.

35. Hancox RJ, Milne BJ, Poulton R. Association between child and adolescent television viewing and adult health: a longitudinal birth cohort study. Lancet. 2004;364:257262.

36. Kerner MS, Kalinski M, Kurrant AB, Small E, Spatz E, Gropack S. Leisure-time internet use does not correlate with physical activity or physical fitness level of ninth grade African-American girls. Pediatr Exerc Sci. 2001;13:402-412.

37. Carnethon MR, Gulati M, Greenland P. Prevalence and cardiovascular disease correlates of low cardiorespiratory fitness in adolescents and adults. JAMA. 2005;294:29812988.

38. Janssen I, Cramp WC. Cardiorespiratory fitness is strongly related to the metabolic syndrome in adolescents. Diabetes Care. 2007;30:2143-2144.

39. Ekelund U, Anderssen SA, Froberg K, Sardinha LB, Andersen LB, Brage S. Independent associations of physical activity and cardiorespiratory fitness with metabolic risk factors in children: the European youth heart study. Diabetologia. 2007;50:1832-1840.

40. Pate RR, Wang CY, Dowda M, Farrell SW, O’Neill JR. Cardiorespiratory fitness levels among US youth 12 to 19 years of age: findings from the 1999-2002 National Health and Nutrition Examination Survey. Arch Pediatr Adolesc Med. 2006;160:1005-1012.

41. Ortega FB, Ruiz JR, Castillo MJ, Sjöström M. Physical fitness in childhood and adolescence: a powerful marker of health. Int J Obes (Lond). 2008;32(1):1-11. 